Saudi Journal of Medicine

Abbreviated Key Title: Saudi J Med ISSN 2518-3389 (Print) |ISSN 2518-3397 (Online)

\title{
Elderly Patients' Adherence, Knowledge and Belief to Medications in Primary Healthcare Centers in Baghdad
}

\author{
Alaa A. Salih ${ }^{1 *}$, Rasha M. Ismail ${ }^{2}$ \\ ${ }^{1}$ Assistant Professor /Family and Community medicine -El Mustansiriyah University College of Medicine \\ ${ }^{2}$ Family physician -Ministry of Health Iraq
}

DOI: $10.36348 / \mathrm{sjm} .2022 . v 07 i 01.002$

| Received: 30.11.2021 | Accepted: 02.01.2022 | Published: 15.01.2022

*Corresponding Author: Alaa A. Salih

Assistant Professor /Family and Community medicine -El Mustansiriyah University College of Medicine

\section{Abstract}

Background: currently the Elderly population is rapidly increasing and with this increment comes more challenges for the healthcare system, non-adherence to medications especially in elderly can lead to economic and human lost that is avoidable by improving patients' compliance and address the related issues leading to the non-adherence. Objectives: 1assess the level of adherence knowledge and belief to medications. 2-identify the related factors causing the nonadherence. Methodology: A cross sectional study was conducted in primary healthcare centers in Baghdad targeting geriatric age group of both genders attending the primary healthcare centers and having at least one diagnosed chronic disease, the study enrolled 10 healthcare centers from both sides of Baghdad. Patients were directly interviewed and data was collected using a questionnaire. Results: The study showed that $(83.4 \%)$ of the sample $(n=385)$ had low adherence level, this percentage tend to increase as the age increases, no significant association was found with gender variable, and a significant association was found with the age variable. most of the participants has low beliefs level (86\%) and only (7\%) of the sample scored (high) on knowledge level which was significantly associated with adherence level, (13.5\%)of the participants faced high difficulty to adhere to their medication. Conclusions: Elderly patients attending the primary healthcare centers in Baghdad had low level of adherence; most of them did not have enough knowledge about their medications. Patients who scored low level of adherence tend to pay more visits to the healthcare centers.

Keywords: elderly, medications, adherence, Knowledge, belief.

Copyright () 2021 The Author(s): This is an open-access article distributed under the terms of the Creative Commons Attribution 4.0 International License (CC BY-NC 4.0) which permits unrestricted use, distribution, and reproduction in any medium for non-commercial use provided the original author and source are credited.

\section{INTRODUCTION}

Populations around the world are rapidly aging, Aging represents both challenges and opportunities and it will increase demand for primary health care and long term medical care $\left.{ }^{\mathrm{i}}\right]$.

The population aged 60 and more is growing each year, by the year 2025 the world will have 1.2 billion people aged 60 and over rising to 1.9 billion in 2050 , while the proportion of elderly population to total population was $5.8 \%$ in 2000 [ii].

Elderly related demographic data In Iraq showed that the proportion of population aged 60 and above increased from $2.8 \%$ in 1950 to $4.2 \%$ in 1975 and then it decreased to $3.4 \%$ in 2010 and for those 65 years and over represent 3.55\% (male632, 753/female794, 489) of total Iraqi population in 2018 and it is expected to reach $8.7 \%$ by year 2025 and $15 \%$ by $2050\left[{ }^{\mathrm{iii}},{ }^{4}\right]$.
Chronological definition of elderly or aged is commonly used, but contested UN tabulations provided figures for both 60 and 65 years of age and older the WHO uses categories starting at the age of 65 and 80 $\left[{ }^{5}\right]$.

Iraq defines the senior citizens as "people who are 60-65 years and above, 60 years for males and 59 for females $\left[{ }^{6}\right]$.

The demographic shift in elderly population is not only a challenge itself, but also because the development of chronic diseases is often associated with and more common among older age groups, these groups will need considerable support from society in the future due to that they have to live with and among chronic conditions over the long term, for the rest of their lives $\left[{ }^{7}\right]$. Chronic diseases are generally considered physical or mental conditions that last more than a year and require ongoing care. They compromise the 
Alaa A. Salih \& Rasha M. Ismail.; Saudi J Med, Jan, 2022; 7(1): 4-14

individuals' physical and social function, the health related quality of life and economic sustainability of healthcare system $\left[{ }^{8]}\right.$.

The most common of the chronic diseases are cardiovascular disease, diabetes, cancer and chronic respiratory disease, together they account for 41 million deaths in the world every year, and this number is increasing $\left[{ }^{7}\right]$.

The combined cost savings from the health and productivity that results from a small reduction in the prevalence of chronic disease cannot be ignored, resulting in a genuine return on investment in a very small span of time, the need for long -term care for the chronic conditions can lead to decline in the QoL of elderly and by recognizing their needs and constraints regarding the chronic diseases would fix many problems the patients face and result in an improved QoL , safety, and overall health in elderly , their productivity will rise as well thereby contributing to the economic and social opportunities $\left[8,{ }^{9}\right]$, so adherence to long term medications can play a crucial part in the process as adherence-where prescribed medications are taken at the right doses and frequency recommended by the healthcare provider-has been shown to improve health outcomes and reduce healthcare costs $\left[{ }^{10}\right]$.

A multifactorial phenomenon factors as age, gender, socioeconomic status and level of disease severity all have shown to affect adherence. Medication non-adherence is recognized as a worldwide public health problem with important implications for the management of chronic diseases and consequent polypharmacy as it is simultaneous and chronic use of multiple medications, predisposing those practicing it to low adherence $\left[{ }^{11}\right]$ it is a major cause of Morbidity especially in elderly patients approximately $10 \%-30$ of hospitalizations maybe result of medication nonadherence, There are many factors that increase the level of non-adherence in elderly age group include adverse drug reactions that come with polypharmacy, decreased visibility and motor dexterity, and decreased autonomy $\left[{ }^{12}\right]$.

Older adults can experience age-related declines in the cognitive processes necessary for medication adherence, and therefore they may be at higher risk for neglecting to take medication as prescribed. This risk is accentuated for individuals suffering from chronic diseases $\left[{ }^{13}\right]$.

Several Classifications had been recognized regarding non-adherence, intentional and nonintentional adherence is two Types of non-adherence behaviors. Intentional non-adherence is a behavior driven by a decision not to take medicines. The drivers of this decision are complex but have been suggested to be based on beliefs, personal circumstances, interpretations of healthcare advice and personal motivation. Unintentional non-adherence reflects person's ability and skill at medicine taking including forgetting, poor manual dexterity, losing medicines or no being able to afford them $\left[{ }^{14}\right]$.

Other studies classify adherence as either primary or secondary. Primary non-adherence is the frequency with which patients fail to fill prescriptions when new medications are started so it is related to refilling and initiation of medication therapy. Secondary non-adherence is defined as the medication being not taken as prescribed when prescriptions are filled. It does not only affect the clinical outcome, but also affect the financial outcome of health system $\left[{ }^{15}\right]$.

According to WHO, where there are multiple factors leading to poor medication adherence, normally classified into five categories: socioeconomic factors, therapy-related factors, patients -related factors, condition related factor, and healthcare system/health care team-related factors $\left[{ }^{16}\right]$.

A patient's medication knowledge is an important factor that affect the medication adherence as it is defined the awareness of the drug name, purpose, administration schedule, adverse or side effects and special administration instructions. Poor medication knowledge can have a negative impact on medication adherence_ especially in elderly_ which may result in increased use of medical resources, such as physician visits, laboratory tests, E.R. Visits And hospital admissions $\left[{ }^{17}\right]$. In general inadequate medicationadherence is the single most modifiable aspect of chronic diseases management $\left[{ }^{18}\right]$. Most studies have focused on improving adherence to one drug group, and thus have limited the applicability to the older population who commonly use multiple medications $\left[{ }^{19}\right]$.

\section{OBJECTIVES}

- Determination of the adherence levels in elderly patients in Baghdad-Iraq

- Determination of the association of the levels of adherence with sociodemographic variables and other medical variables.

- Exploration some of the difficulties elderly patients face that hinder proper adherence.

\section{PATIENTS AND METHODS}

The study designed as cross sectional, the data collected in primary health care centers in Baghdad city including six primary healthcare centers from $\mathrm{AL}$ KARKH district and four from AL-RUSAFA district, the patients were directly interviewed for 7-10 minutes each in the waiting hall of the primary center, data were collected approximately 3 days a week, researcher explained the aim of the study and details of the questionnaire. 
Alaa A. Salih \& Rasha M. Ismail.; Saudi J Med, Jan, 2022; 7(1): 4-14

A convenient sample size was collected, the sample included all patients aging equal or more than 65 years (according to the WHO definition of geriatric age group), both genders with least one chronic disease who were present at the time of the study and willing to participate.

A questionnaire used MMAS- 8 scale of medication adherence to assess the adherence ,patients were categorized into three groups: low adherence(MMAS $<6$ ), medium adherence(MMAS 6$<8)$ and high adherence(MMAS=8) [26], which is also used for beliefs ( 7 points high), $(<7->5$ points is medium) and ( $<=5$ points is low), knowledge( 6 point is high), $(<6->4$ is medium $)$ and $(<=4$ is low $)$ and difficulty(5points is high), $(<5->3$ is medium) and $(<=3$ is low) $\left[{ }^{20}\right]$. The adherence question included dichotomous responses (YES/NO).

\section{The questionnaire consisted of two parts}

A -Part one: sociodemographic data and medical variables: including

- Age

- Gender

- Marital status

- Level of education

- Social habits

- Number of chronic diseases

- Number of medications taken.
- Number. of visits to the PHC per month

- Number of family members living in the house.

B-Part two: assessment of the factors associated with low adherence to pharmacotherapy in elderly patients.

Ethical Issue: verbal consent was taken from each individual after full explanation of the aim and the method of the research. Ensure to the individual there was no harm or any invasion of privacy or dignity. Informed the subject about the methods will be used to protect anonymity and confidentiality

\section{STATISTICAL ANALYSIS}

Analysis of data was carried out using the available statistical package of SPSS-27 (Statistical Packages for Social Sciences- version 27). Data were presented in frequency and percentage.

The significance of difference of different percentages was tested using Pearson Chi-square test $(\chi 2$-test) with application of Yate's correction or Fisher Exact test whenever applicable. Statistical significance was considered whenever the $\mathrm{P}$ value was equal or less than $0.05\left[{ }^{21}\right]$.

\section{RESULTS}

Table-1: The sociodemographic and medical variables of the studied sample. $(n=385)$

\begin{tabular}{|l|l|l|l|}
\hline \multirow{5}{*}{ Age (years) } & & No & \% \\
\hline \multirow{5}{*}{ Gender } & $65---69$ & 154 & 40 \\
\cline { 2 - 4 } & $70---74$ & 99 & 25.7 \\
\cline { 2 - 4 } & $75---79$ & 69 & 17.9 \\
\cline { 2 - 4 } & $80---84$ & 53 & 13.8 \\
\cline { 2 - 4 } & $=>85$ years & 10 & 2.6 \\
\hline Larital status & Male & 191 & 49.6 \\
\hline \multirow{5}{*}{ Level of education } & Female & 194 & 50.4 \\
\cline { 2 - 4 } & Married & 250 & 64.9 \\
\cline { 2 - 4 } & Single & 44 & 11.4 \\
\cline { 2 - 4 } & Widowed & 91 & 23.6 \\
\hline \multirow{5}{*}{ Employment status } & No education & 48 & 12.5 \\
\cline { 2 - 4 } & Primary & 58 & 15.1 \\
\cline { 2 - 4 } & Intermediate & 71 & 18.4 \\
\cline { 2 - 4 } & Secondary & 102 & 26.5 \\
\cline { 2 - 4 } & Graduate & 83 & 21.6 \\
\cline { 2 - 4 } & Postgraduate & 23 & 6 \\
\hline Sumber of chronic diseases & Unemployed & 116 & 30.1 \\
\cline { 2 - 4 } & Retired & 154 & 40 \\
\cline { 2 - 4 } & Freelancer & 70 & 18.2 \\
\cline { 2 - 4 } & Employed & 45 & 11.7 \\
\hline Social habits-Smoking & Smoking & 107 & 27.8 \\
\cline { 2 - 4 } & None & 278 & 72.2 \\
\hline \multirow{3}{*}{ Social habits-Alcohol } & Alcohol & 22 & 5.7 \\
\cline { 2 - 4 } & None & 363 & 94.3 \\
\hline & One & 97 & 25.3 \\
\cline { 2 - 4 } & Two & 174 & 45.4 \\
\hline & & & \\
\hline
\end{tabular}


Alaa A. Salih \& Rasha M. Ismail.; Saudi J Med, Jan, 2022; 7(1): 4-14

\begin{tabular}{|l|l|l|l|}
\hline \multirow{4}{*}{ Number of medication taken } & Three & 94 & 24.5 \\
\cline { 2 - 4 } & Four \& more & 18 & 4.7 \\
\hline \multirow{5}{*}{ Frequency of use of medical services } & $1---3$ & 135 & 35.1 \\
\cline { 2 - 4 } & $4---6$ & 170 & 44.2 \\
\cline { 2 - 4 } & $7---9$ & 74 & 19.2 \\
\cline { 2 - 4 } & $=>10$ items & 6 & 1.6 \\
\cline { 2 - 4 } & DNK & 127 & 33 \\
\cline { 2 - 4 } & $1---3$ & 131 & 34 \\
\cline { 2 - 4 } & $4---6$ & 75 & 19.5 \\
\cline { 2 - 4 } & $>6$ months & 52 & 13.5 \\
\hline & 1 memebr & 8 & 2.1 \\
\cline { 2 - 4 } & $2---3$ & 126 & 32.7 \\
\cline { 2 - 4 } & $4---5$ & 158 & 41 \\
\cline { 2 - 4 } & $>5 m e m b e r s$ & 93 & 24.2 \\
\hline
\end{tabular}

Table-2: Responses to memory (adherence) questions. $(n=385)$

\begin{tabular}{|c|c|c|c|c|}
\hline \multirow[t]{2}{*}{ A-Memory (Adherence): } & \multicolumn{2}{|l|}{ Yes } & \multicolumn{2}{|l|}{ No } \\
\hline & No & $\%$ & No & $\%$ \\
\hline Sometimes forget to take medications for a day or two & 265 & 68.8 & 120 & 31.2 \\
\hline There are days that totally forget to take medicine & 218 & 56.6 & 167 & 43.4 \\
\hline Forget to take medicine with him/her upon leaving home & 168 & 43.6 & 217 & 56.4 \\
\hline Take his/her pills by him/herself & 295 & 76.6 & 90 & 23.4 \\
\hline $\begin{array}{l}\text { Ever stopped taking medicine because he/she did not buy it, or no one } \\
\text { bought it for him/her }\end{array}$ & 158 & 41.0 & 227 & 59.0 \\
\hline Remember the names of his/her medicines & 197 & 51.2 & 188 & 48.8 \\
\hline Remember the timing of each medicine intake & 246 & 63.9 & 139 & 36.1 \\
\hline Use an alarm or a person to help him/her remember his/her medications & 239 & 62.1 & 146 & 37.9 \\
\hline
\end{tabular}

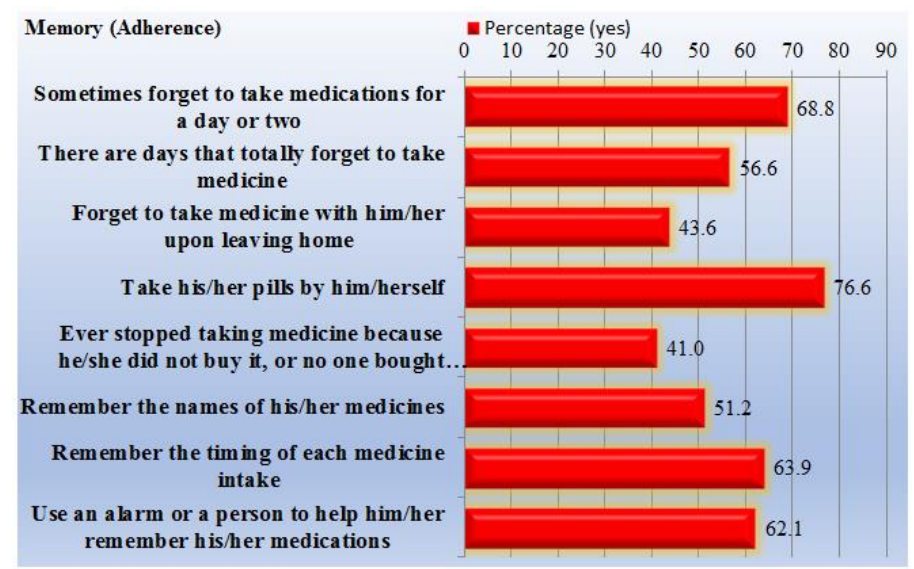

Fig-1: Responses of the sample to memory (adherence) questions. $(n=385)$

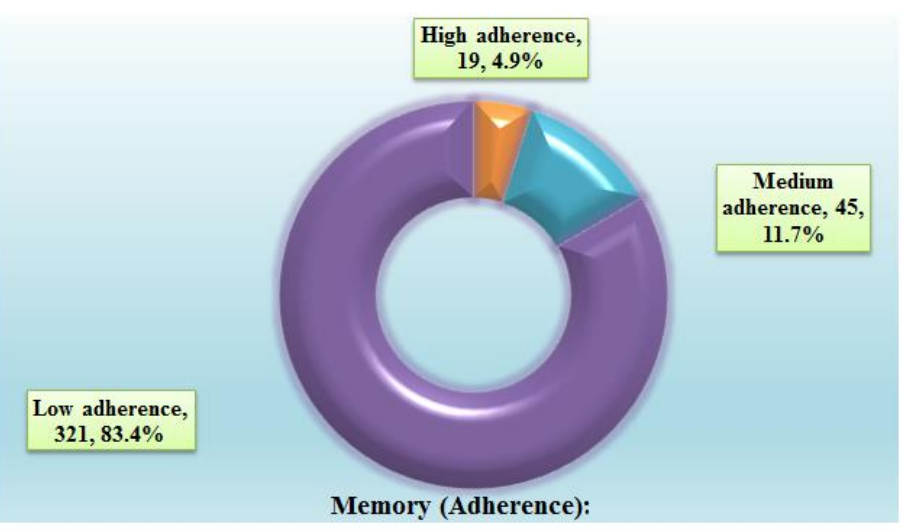

Fig-2: Shows the level of adherence among the studied sample. $(n=385)$ 


\section{Beliefs}

Table-3: Responses of the sample to (Beliefs) questions. $(n=385)$

\begin{tabular}{|c|c|c|c|c|}
\hline \multirow[t]{2}{*}{ B-Beliefs: } & \multicolumn{2}{|l|}{ Yes } & \multicolumn{2}{|l|}{ No } \\
\hline & No & $\%$ & No & $\%$ \\
\hline Ever stopped taking your medicine because he/she did not feel better & 266 & 69.1 & 119 & 30.9 \\
\hline Ever stopped taking his/her medicine because he/she felt better & 211 & 54.8 & 174 & 45.2 \\
\hline Change the doses of medications without the physician consultation & 144 & 37.4 & 241 & 62.6 \\
\hline Believe that taking medications is not harmful & 195 & 50.6 & 190 & 49.4 \\
\hline Think the prices of his/her medications are high & 269 & 69.9 & 116 & 30.1 \\
\hline The price of his/her medications affect the purchase of them & 133 & 34.5 & 252 & 65.5 \\
\hline The method of drug intake prevents him/her from taking it & 158 & 41.0 & 227 & 59.0 \\
\hline
\end{tabular}

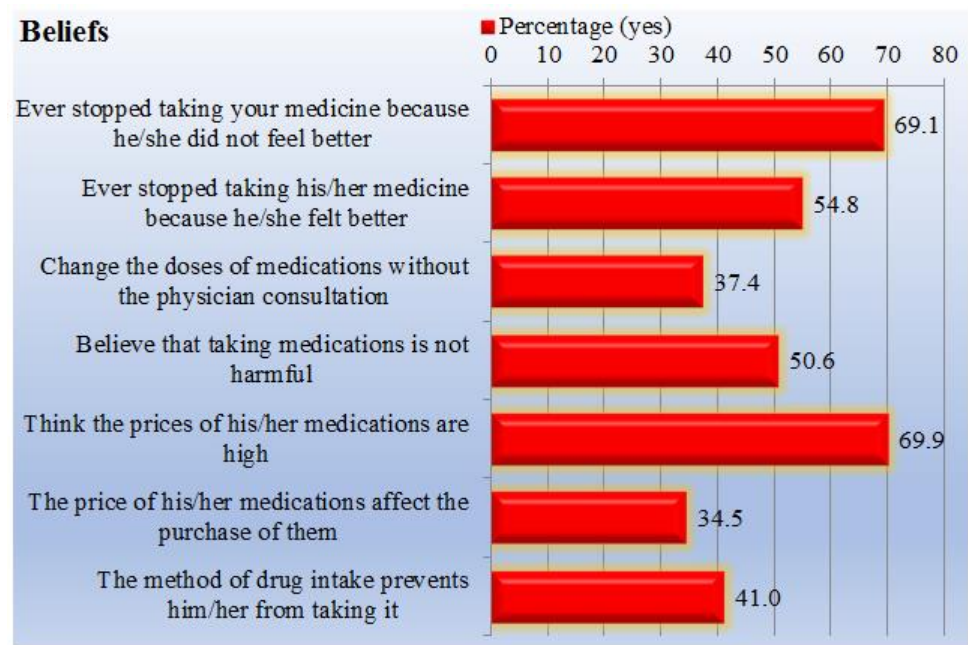

Fig-3: Shows the distribution of the answers to (Beliefs) questions.

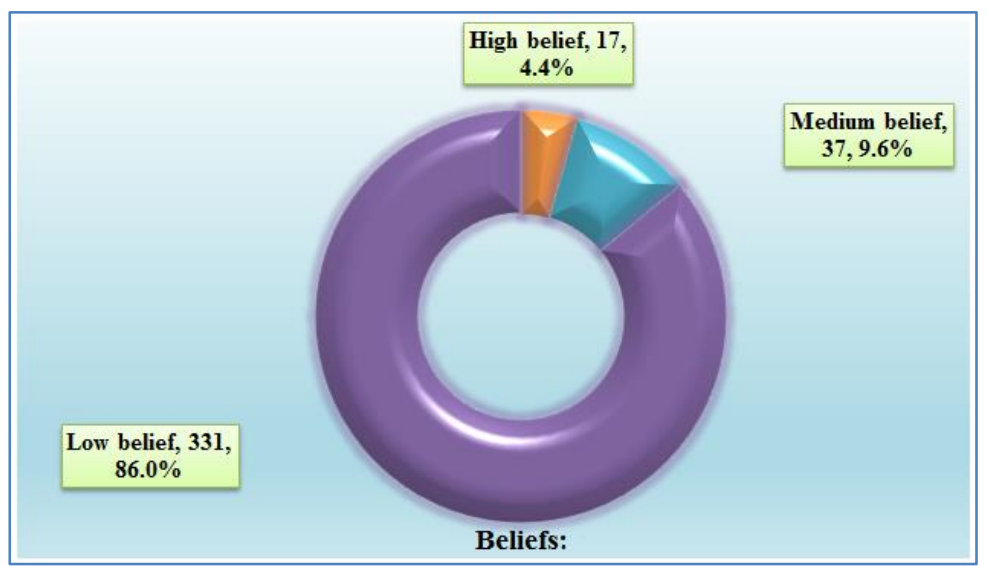

Fig-4: Shows the level of Beliefs among the studied sample. $(n=385)$

\section{Knowledge}

Table-4: The responses to General Knowledge of medications questions. $(n=385)$

\begin{tabular}{|l|l|l|l|l|}
\hline C-General Knowledge: & Yes & \multicolumn{1}{l|}{ No } \\
\cline { 2 - 5 } & No & $\mathbf{\%}$ & No & $\mathbf{\%}$ \\
\hline Have enough knowledge about his/her medications & 268 & 69.6 & 117 & 30.4 \\
\hline Read the medicine leaflet or ask someone to read it for him/her & 212 & 55.1 & 173 & 44.9 \\
\hline Ask the physician about the medicine's side effects before taking it & 199 & 51.7 & 186 & 48.3 \\
\hline Take medications randomly without a physician prescription & 157 & 40.8 & 228 & 59.2 \\
\hline Think the continuous use of medications will prevent further deterioration & 294 & 76.4 & 91 & 23.6 \\
\hline Would continue to take the medications despite the side effects & 206 & 53.5 & 179 & 46.5 \\
\hline
\end{tabular}




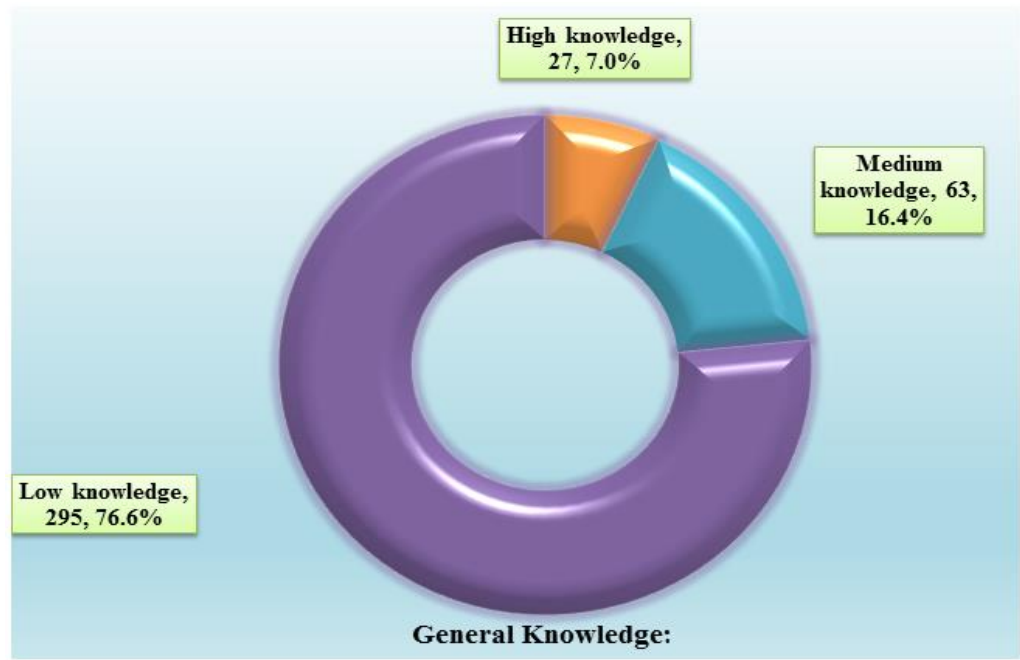

Fig-5: The level of general knowledge among the studied sample

\section{Difficulty}

Table-5: the responses to difficulty to adherence questions. $(n=385)$

\begin{tabular}{|l|l|l|l|l|l|l|}
\hline D-Difficulty: & Easy & \multicolumn{2}{l|}{ With difficulty } & \multicolumn{2}{l|}{ Very difficult } \\
\cline { 2 - 7 } & No & $\mathbf{\%}$ & No & \% & No & \% \\
\hline How it is to read the prescription leaflet & 64 & 16.6 & 253 & 65.7 & 68 & 17.7 \\
\hline How it is to open the medicine container & 301 & 78.2 & 76 & 19.7 & 8 & 2.1 \\
\hline How it is to remember taking your medications & 139 & 36.1 & 229 & 59.5 & 17 & 4.4 \\
\hline How it is to commit to the time of each medication & 133 & 34.5 & 225 & 58.4 & 27 & 7.0 \\
\hline $\begin{array}{l}\text { How it is to take a lot of medications at the same } \\
\text { time }\end{array}$ & 136 & 35.3 & 201 & 52.2 & 48 & 12.5 \\
\hline
\end{tabular}

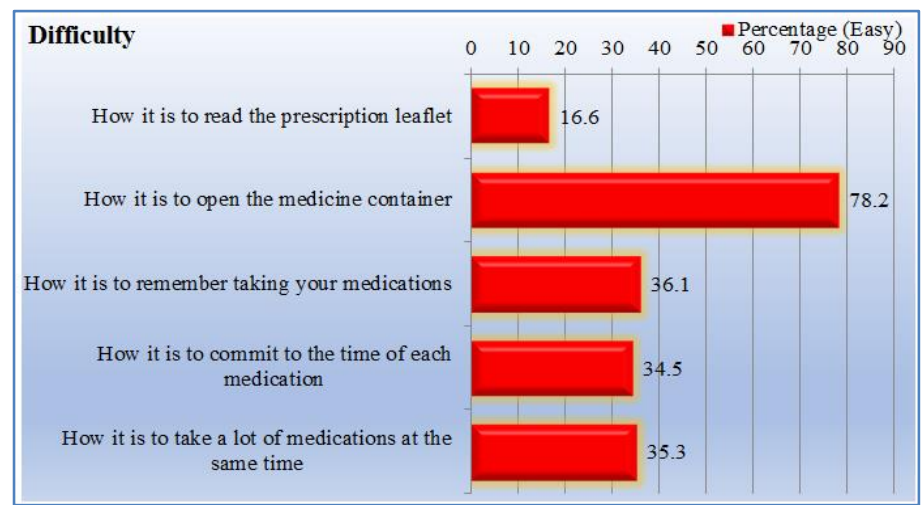

Fig-6: Shows the distribution of the response (easy).

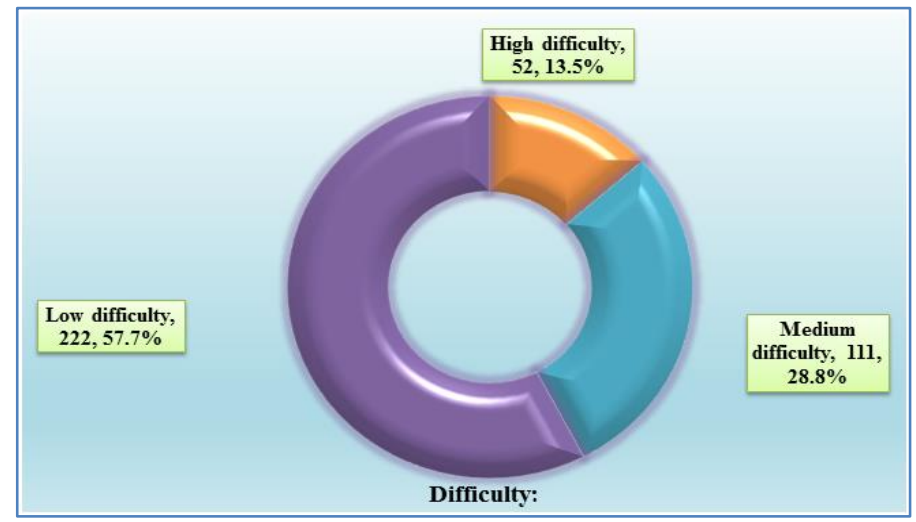

Fig-7: Shows the level of difficulty of adherence among the sample. 


\section{Association of adherence with sociodemographic features}

Table-6: Association the level of adherence with the sociodemographic variables

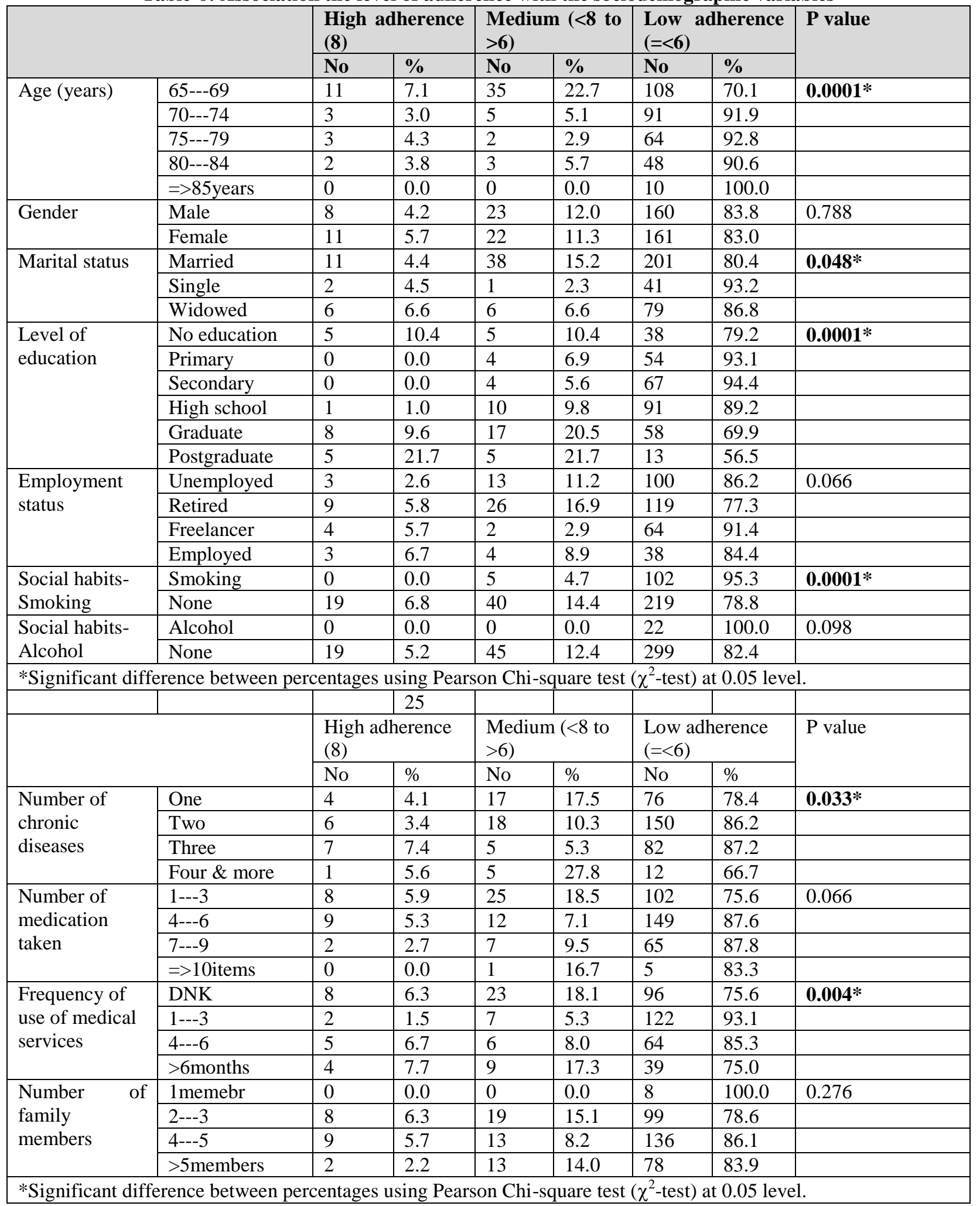


Alaa A. Salih \& Rasha M. Ismail.; Saudi J Med, Jan, 2022; 7(1): 4-14

Table-7: Association of the level of adherence with Beliefs, General knowledge and Difficulty levels

\begin{tabular}{|c|c|c|c|c|c|c|c|c|}
\hline & \multicolumn{2}{|c|}{$\begin{array}{l}\text { High adherence } \\
\text { (8) }\end{array}$} & \multicolumn{2}{|c|}{$\begin{array}{l}\text { Medium }(<8 \text { to } \\
>6)\end{array}$} & \multicolumn{2}{|c|}{$\begin{array}{l}\text { Low adherence } \\
(<6)\end{array}$} & \multirow[t]{2}{*}{$P$ value } \\
\hline & & No & $\%$ & No & $\%$ & No & $\%$ & \\
\hline \multirow[t]{3}{*}{ B-Beliefs: } & High belief (7) & 0 & 0.0 & 7 & 41.2 & 10 & 58.8 & $0.0001 *$ \\
\hline & Medium $(<7$ to $>5)$ & 4 & 10.8 & 15 & 40.5 & 18 & 48.6 & \\
\hline & Low belief $(=<5)$ & 15 & 4.5 & 23 & 6.9 & 293 & 88.5 & \\
\hline \multirow{3}{*}{$\begin{array}{l}\text { C-General } \\
\text { Knowledge: }\end{array}$} & High knowledge (6) & 2 & 7.4 & 9 & 33.3 & 16 & 59.3 & $0.005 *$ \\
\hline & Medium $(<6$ to $>4)$ & 4 & 6.3 & 8 & 12.7 & 51 & 81.0 & \\
\hline & $\begin{array}{l}\text { Low knowledge } \\
(=<4)\end{array}$ & 13 & 4.4 & 28 & 9.5 & 254 & 86.1 & \\
\hline \multirow{3}{*}{$\begin{array}{l}\text { D- } \\
\text { Difficulty: }\end{array}$} & High difficulty (5) & 0 & 0.0 & 0 & 0.0 & 52 & 100.0 & 0.0001* \\
\hline & Medium $(<5$ to $>3)$ & 1 & 0.9 & 6 & 5.4 & 104 & 93.7 & \\
\hline & Low difficulty $(=<3)$ & 18 & 8.1 & 39 & 17.6 & 165 & 74.3 & \\
\hline \multicolumn{9}{|c|}{ *Significant difference between percentages using Pearson Chi-square test ( $\chi^{2}$-test) at 0.05 level. } \\
\hline & & & & 27 & & & & \\
\hline
\end{tabular}

\section{DISCUSSION}

Physicians must be vigilant in the fight against medication non-adherence, and since older adults are usually at highest risk due to multi-comorbidities and polypharmacy often combined in this age group [22]. The world health organization had termed adherence to therapy a "multidimensional phenomenon" comprised from five dimension [23], this study focused on socioeconomic factors, patient-related factors, and therapy-related factors. In the current study, the memory (adherence) for medications was low in $83.4 \%$ of participants and high among only $4.9 \%$, mostly attributed to self-administration of medication (76.6\%), forgetting to take medication of a day or two $(68.8 \%)$ or totally forgetting to take it $(56.6 \%)$ and not knowing the names of their medications $(48.8 \%)$.

The adherence of our patients was much lower compared to results of Awad et al. (2020) in Kuwait, who studied 424 elderly patients from ten PHCCs, and reported that $54.7 \%$ had low adherence, mostly attributed to forgetfulness, missing doses when they feel better, or because of multiple doses (> once daily) [24]. In another study done by Salama et al. (2017) in Egypt, who studied 438 patients, and reported that $73.2 \%$ were considered adherent [25].

In Portugal, a study done by Gomes et al. (2020), enrolling 1089 elderly patients, and reported that $47.7 \%$ showed low adherence which was related to forgetfulness, difficult medication schedules, worrying about adverse effects, and cost issues ${ }^{26}$. The difference of adherence level between this study and the aforementioned studies could be attributed to different methods used to assess the adherence of patients to medication, and the actual lack of a standardized gold standard method.

The beliefs of patients were low in $86 \%$, and that was mostly attributed to high medication cost (69.9\%), stopping medication because they did not feel better $(69.1 \%)$, or the felt better $(54.8 \%)$, and $49.4 \%$ thought taking medication was harmful. The interaction between beliefs about medication and medication burdens (including adherence) has very influential role in determining the outcome of patients, as reported by Mohmmed et al. (2016) [27].

In this study, $76.6 \%$ of patients had low knowledge about medications, as $76.4 \%$ of them think they should continuously use medication to prevent disease progression, and $69.6 \%$ of them had enough knowledge about their medications, and as shown in Table 6; higher level of education was associated with better medication adherence. Jin et al. (2016) in Korea had studied factors related to medication adherence, and reported that patients' satisfaction, sufficient explanation of medication counseling, education level, health-related problems, and dosing frequency [28], and this highlighted a very pivotal point in patients doctor relationship, as the physicians are ought to provide sufficient explanation about patients illness and the appropriate medication they prescribe for each, building trust and improving adherence.

An important point is education of patients about their diseases, regardless of their backgrounds, especially for chronic diseases that some of them may not understand the reasons for taking the medication [29]. This was also studied by Taibanguay et al. (2019) in Thailand, and reported that education patients with rheumatoid arthritis significantly increased patients' adherence to treatment [30]. In the current study, $13.5 \%$ of patients had high medication difficulty, and it was probably due to the very low proportion of patient who knows how to read the prescription $(16.6 \%)$, and how to remember the timing, or commit to it, and taking a lot of medication at the same time.

These results were in concordance with report of Pantuzza et al. (2017), in their extensive systematic review of 35 studies that investigated the association between complexity in regimens and adherence of medication and in 28 of those studies high complexity 
Alaa A. Salih \& Rasha M. Ismail.; Saudi J Med, Jan, 2022; 7(1): 4-14

was associated with lower adherence level [31]. Medication factors should always be put in mind while treating patients with multimorbidity, as the guidelines for separated diseases are not inherently designed for treating patients with combined disease, which would ultimately leads to higher burden on patients, linked with lower patients satisfaction and higher disruption to their lives ${ }^{32}$.In this study, patients older than 65 years were enrolled, the most frequent age group was 65-69 years $(40 \%)$, then $70-74$ years $(25.7 \%)$, and older age was related to lower medication adherence and lower belief These results were in comparison to results of Awad et al. (2020) in Kuwait, who reported that $73.1 \%$ of participants were aged between 65 and 74 years, and those aged 75 years and older had higher medication related burden and higher difficulty living with medications $\left[{ }^{24}\right]$.

Tsai et al. in Taiwan studied 193 elderly patients with a mean age of 76.2 years, and reported no significant difference in mean age between low and high medication adherence [33]. With advancing age, there are increasing number of factors affecting welfare, living style, and health behavior, however GautérioAbreu in Brazil (2015) reported that the desire to feel better and healthy was enough to make elder patient have better adherence to medications [34].

In this study, gender was not associated with adherence level, while married individuals showed better adherence level compared to unmarried and widowed patients. These results were consistent with results of Mahmoodi et al. (2019) in Iran, who studied 455 older adults regarding medication adherence, and reported that $54.5 \%$ of them had low adherence level, and this was not associated with gender, but married individuals had higher adherence level [35].

While Manteuffel et al. (2014) reported that women had lower adherence than men regarding chronic disease medication like diabetes mellitus and hypertension 36], also Biffi et al. (2020) reported that older than 65 year-women had lower adherence to antihypertensive medications [37] as Joung et al. (2020) reported that women are more likely to develop sideeffects with newer anti-diabetic agents much more likely than men [38]. Regarding marital status, Wu et al. (2012) reported that unmarried patients had lower medication adherence are twice the risk for having cardiac events ${ }^{39}$. One aspect could be related to loneliness, as Kusaslan (2018) in Turkey reported that, loneliness was correlated with lower adherence to medication [40].

In the current study, smoking was associated with lower medication adherence, and although alcohol consumption showed no significant association with adherence, all alcoholics had low adherence. This was consistent with results of Aggarwal and Mosca (2010) in India, who reported that smoking was significantly associated with lower adherence level of antihypertensive and lipid lowering agents [41]. Fialová et al. (2018) in Czech Republic reported that alcohol is one of the psychological/behavioral factors that are associated with medical poor adherence [42]. One explanation might be the effect of smoking habit on daily activities and routine of patients, negatively affecting medication adherence. On the other hand, alcoholics could think that alcohol would interact with the medications they should take [43].

In the current study, higher number of chronic diseases was associated with better adherence, while the number of medications was not related. With aging, the number of health events and chronic diseases usually increase, accompanied by an increment in number of medications to control these diseases [44], leading to inaccurate use of medications and a higher incidence of adverse events, which could be avoided by appropriate use of medications [45]. The prevalence of poor adherence in patients taking high number of medications (polypharmacy) ranges from $6 \%$ to $55 \%$, which could be due to the difference in defining polypharmacy [46].

Frequency of medical services utilization was inversely associated with medication adherence. Aging population requires more healthcare services, suffers more inpatients admissions with longer be times than any other age groups, thus enforcing more burden on healthcare [47]. However, one explanation for this contradiction is that patients with poor medical adherence are at more risk for hospitalization, which indirectly point that patients who are compliant to therapy have lower utilization for healthcare services [48].

\section{CONCLUSIONS}

1. Low adherence to medications is alarming among the elderly patients in Baghdad city.

2. Patients have poor knowledge regarding their medications on many levels.

3. The level of adherence decreases with getting older in age.

4. Patients with low adherence level attend more frequently to PHCs causing more time spent from healthcare providers.

\section{REFERENCES}

1. Www.who.int/ageing/en/ accessed at 23rd July 2020.

2. WHO/EMR. Technical paper: health care of elderly in the eastern mediterranean region: challenges and prospectives.

3. Hussain, H. Y. (2016). Elderly, health and sociodemographic profile in Iraq, the context of conflict, violence and social exclusion, systematic review. Middle East Journal of Age and Ageing, 83(4013), 1-8. 
Alaa A. Salih \& Rasha M. Ismail.; Saudi J Med, Jan, 2022; 7(1): 4-14

4. Www.indexmundi.com/Iraq/demographics_profile. html accessed at 23rd of July 2020.

5. WHO technical paper: Men ageing and Health, 01/NHM/NPH/01-2 accessed from http://apps.who.int/iris/bitstream/handle/10665/669 41/WHO_NMH_NPH_01.2.pdf. At $23^{\text {rd }}$ of July 2020 .

6. Jamil, N. F., Salih, A. A., \& Razzaq, D. I. (2019). Mental Health Assessment of Elderly People Attending Geriatric Clinic in Medical City. Open Journal of Psychiatry, 9(2), 98-106.

7. Georgsson, M. (2018). An aging population, larger chronic disease burden, and reliance on digital selfmanagement tools require contributions from nurse informaticians. On-Line Journal of Nursing Informatics, 22(3).

8. Fernandez-Lazaro, C. I., García-González, J. M., Adams, D. P., Fernandez-Lazaro, D., MielgoAyuso, J., Caballero-Garcia, A., \& Miron-Canelo, J. A. (2019). Adherence to treatment and related factors among patients with chronic conditions in primary care: a cross-sectional study. BMC family practice, 20(1), 1-12.

9. Maresova, P., Javanmardi, E., Barakovic, S., Husic, J. B., Tomsone, S., Krejcar, O., \& Kuca, K. (2019). Consequences of chronic diseases and other limitations associated with old age-a scoping review. BMC public health, 19(1), 1-17.

10. Smaje, A., Weston-Clark, M., Raj, R., Orlu, M., Davis, D., \& Rawle, M. (2018). Factors associated with medication adherence in older patients: a systematic review. Aging Medicine, 1(3), 254-266.

11. Roy, N. T., Sajith, M., \& Bansode, M. P. (2017). Assessment of Factors Associated with Low Adherence to Pharmacotherapy in Elderly Patients. Journal of Young Pharmacists, 9(2).

12. Park, H.Y., Seo, Sa., Yoo, H. (2018). Medication adherence and beliefs about medication in elderly patients living alone with chronic diseases, Dovepress $24^{\text {th }}$. Jan. 2018.

13. Levy, A. E., Huang, C., Huang, A., \& Ho, P. M. (2018). Recent approaches to improve medication adherence in patients with coronary heart disease: Progress towards a learning healthcare system. Current atherosclerosis reports, 20(1), 1-9.

14. Insel, K., Morrow, D., Brewer, B., \& Figueredo, A. (2006). Executive function, working memory, and medication adherence among older adults. The Journals of Gerontology Series B: Psychological Sciences and Social Sciences, 61(2), P102-P107.

15. Stack, R. J., Bundy, C. E., Elliott, R. A., New, J. P., Gibson, M., \& Noyce, P. R. (2010). Intentional and unintentional non-adherence in community dwelling people with type 2 diabetes: the effect of varying numbers of medicines. The British Journal of Diabetes \& Vascular Disease, 10(3), 148-152.

16. Wai, Y. L. (2015). Paula Fresco medication adherence measures: an overview. Biomed research international volume 2015 article ID 21704712 pages.
17. Sabate, E. (2003). Adherence to long -term therapies: evidence for action, World Health Organization, Geneva, Switzerland.

18. Chan, F. W. K., Wong, F. Y. Y., So, W. Y., Kung, K., \& Wong, C. K. M. (2013). How much do elders with chronic conditions know about their medications?. BMC geriatrics, 13(1), 1-7.

19. Ulley, J., Harrop, D., Ali, A., Alton, S., \& Davis, S. F. (2019). Deprescribing interventions and their impact on medication adherence in communitydwelling older adults with polypharmacy: a systematic review. BMC geriatrics, 19(1), 1-13.

20. https://www.sralab.org/rehabilitationmeasures/morisky-medication-adherence-scale8Accessed at 10.2.2021.

21. Biostatistics. (2013). A Foundation for Analysis in the Health Sciences. Wayne W Daniel \& Chad L Cross; $10^{\text {th }}$ ed. John Wiley \& Sons Inc, USA.

22. Meyer, L. (2015). Improving Medication Adherence. Today's Geriatric Medicine. 2015;8(1):12.

23. Alvi, Y., Najam Khalique, A. A., Khan, H. S., \& Faizi, N. (2019). World Health Organization dimensions of adherence to antiretroviral therapy: A study at antiretroviral therapy centre, Aligarh. Indian journal of community medicine: official publication of Indian Association of Preventive \& Social Medicine, 44(2), 118.

24. Awad, A., Alhadab, A., \& Albassam, A. (2020). Medication-Related Burden and Medication Adherence Among Geriatric Patients in Kuwait: A Cross-Sectional Study. Frontiers in Pharmacology, 11.

25. Salama, A. A., Yasin, A. E. R. A., \& Elbarbary, W. (2017). Medication knowledge as a determinant of medication adherence in geriatric patients, Serse Elian City, Menoufia Governorate, Egypt. Menoufia Medical Journal, 30(1), 63.

26. Gomes, D., Placido, A. I., Mó, R., Simões, J. L., Amaral, O., Fernandes, I., ... \& Roque, F. (2020). Daily medication management and adherence in the polymedicated elderly: A cross-sectional study in Portugal. International journal of environmental research and public health, 17(1), 200.

27. Mohammed, M. A., Moles, R. J., \& Chen, T. F. (2016). Medication-related burden and patients' lived experience with medicine: a systematic review and metasynthesis of qualitative studies. BMJ open, 6(2), e010035.

28. Jin, H., Kim, Y., \& Rhie, S. J. (2016). Factors affecting medication adherence in elderly people. Patient preference and adherence, 10, 2117.

29. Yap, A. F., Thirumoorthy, T., \& Kwan, Y. H. (2016). Medication adherence in the elderly. Journal of Clinical Gerontology and Geriatrics, 7(2), 64-67.

30. Taibanguay, N., Chaiamnuay, S., Asavatanabodee, P., \& Narongroeknawin, P. (2019). Effect of patient education on medication adherence of 
Alaa A. Salih \& Rasha M. Ismail.; Saudi J Med, Jan, 2022; 7(1): 4-14

patients with rheumatoid arthritis: a randomized controlled trial. Patient preference and adherence, 13, 119.

31. Pantuzza, L. L., Ceccato, M. D. G. B., Silveira, M. R., Junqueira, L. M. R., \& Reis, A. M. M. (2017). Association between medication regimen complexity and pharmacotherapy adherence: a systematic review. European journal of clinical pharmacology, 73(11), 1475-1489.

32. Ong, K. Y., Lee, P. S. S., \& Lee, E. S. (2020). Patient-centred and not disease-focused: a review of guidelines and multimorbidity. Singapore medical journal, 61(11), 584.

33. Tsai, K. T., Chen, J. H., Wen, C. J., Kuo, H. K., Lu, I. S., Chiu, L. S., ... \& Chan, D. C. (2012). Medication adherence among geriatric outpatients prescribed multiple medications. The American journal of geriatric pharmacotherapy, 10(1), 6168.

34. Gautério-Abreu, D. P., Santos, S. S. C., Silva, B. T., Ilha, S., \& Gomes, G. C. (2015). Elderly receiving outpatient care: reasons for adherence/nonadherence to medication. Texto \& Contexto-Enfermagem, 24, 1094-1103.

35. Mahmoodi, H., Nahand, F. J., Shaghaghi, A., Shooshtari, S., Jafarabadi, M. A., \& Allahverdipour, H. (2019). Gender based cognitive determinants of medication adherence in older adults with chronic conditions. Patient preference and adherence, 13, 1733.

36. Manteuffel, M., Williams, S., Chen, W., Verbrugge, R. R., Pittman, D. G., \& Steinkellner, A. (2014). Influence of patient sex and gender on medication use, adherence, and prescribing alignment with guidelines. Journal of women's health, 23(2), 112-119.

37. Biffi, A., Rea, F., Iannaccone, T., Filippelli, A., Mancia, G., \& Corrao, G. (2020). Sex differences in the adherence of antihypertensive drugs: a systematic review with meta-analyses. $B M J$ open, 10(7), e036418.

38. Joung, K. I., Jung, G. W., Park, H. H., Lee, H., Park, S. H., \& Shin, J. Y. (2020). Gender differences in adverse event reports associated with antidiabetic drugs. Scientific reports, 10(1), 1-10.

39. Wu, J. R., Lennie, T. A., Chung, M. L., Frazier, S. K., Dekker, R. L., Biddle, M. J., \& Moser, D. K. (2012). Medication adherence mediates the relationship between marital status and cardiac event-free survival in patients with heart failure. Heart \& Lung, 41(2), 107-114.

40. Kusaslan Avci, D. (2018). Evaluation of the relationship between loneliness and medication adherence in patients with diabetes mellitus: a cross-sectional study. Journal of International Medical Research, 46(8), 3149-3161.

41. Aggarwal, B., \& Mosca, L. (2010). Lifestyle and psychosocial risk factors predict non-adherence to medication. Annals of Behavioral Medicine, 40(2), 228-233.
42. Fialová, D., Kummer, I., Držaić, M., \& Leppee, M. (2018). Ageism in medication use in older patients. In Contemporary perspectives on ageism (pp. 213240). Springer, Cham.

43. Holton, A. E., Keeney, C., Ryan, B., \& Cousins, G. (2020). Prevalence of potentially serious alcoholmedication interactions in older adults in a community pharmacy setting: a cross-sectional study. BMJ open, 10(8), e035212.

44. Ramos, L. R., Tavares, N. U. L., Bertoldi, A. D., Farias, M. R., Oliveira, M. A., Luiza, V. L., ... \& Mengue, S. S. (2016). Polypharmacy and Polymorbidity in Older Adults in Brazil: a public health challenge. Revista de saude publica, 50.

45. Payne, R. A., Abel, G. A., Avery, A. J., Mercer, S. W., \& Roland, M. O. (2014). Is polypharmacy always hazardous? A retrospective cohort analysis using linked electronic health records from primary and secondary care. British journal of clinical pharmacology, 77(6), 1073-1082.

46. Zelko, E., Klemenc-Ketis, Z., \& Tusek-Bunc, K. (2016). Medication adherence in elderly with polypharmacy living at home: a systematic review of existing studies. Materia socio-medica, 28(2), 129.

47. Marešová, P., Mohelská, H., \& Kuča, K. (2015). Economics aspects of ageing population. Procedia economics and finance, 23, 534-538.

48. Pednekar, P., Heller, D. A., \& Peterson, A. M. (2020). Association of medication adherence with hospital utilization and costs among elderly with diabetes enrolled in a state pharmaceutical assistance program. Journal of Managed Care \& Specialty Pharmacy, 26(9), 1099-1108. 